Editorial

\title{
Drug discovery: science and/or art?
}

\author{
Diego Liberati \\ National Research Council of Italy, Italy
}

${ }^{\star}$ Correspondence to: Diego Liberati, National Research Council of Italy, Italy; E-Mail: diego.liberati@polimi.it

Received: June 09, 2018; Accepted: June 15, 2018; Published: June 16, 2018;

\section{Editorial}

Pharma companies have been among the most profitable ones at least until the new era of the Info companies like Google, but are still quite interesting, and in fact not so different in principle: it is always question of information, a drug is useful to restore the lost info in an organism, trying not to override to much of what it is still working!

One of the reason for such healthy and wealthy attitude is that drug discovery is not obvious nor simple nor cheap nor fast, as a rule: a good chemical could imply a fortune even in the few years of validity of the patent. A thorough knowledge of pathophysiology is required in order to design biochemistry able to heal without damaging to much. Intuition and experience are paramount, but nowadays at least a couple of approaches are keen to help.

On one side, machine learning, able to identify the statistical properties of the known, may be of great help in forecasting at least simple but necessary properties of the candidate drug, like hydrophilic or hydrophobic behavior.

On the other side, esascale high performance computing is nowadays able to assist physical chemists in simulate and forecast the properties of a big molecule taking into account the properties of every single atom composing its structure.

Such approaches, nowadays seen as opposite, probably because inherited by different communities not sharing a common background, are instead keen to be complementary, as often in science and even in life, when almost perfect but still not sufficient great approaches are combined.

The best would of course be to have an algorithm already able to combine both, but this is still a kind of Graal in the minds of the many scientist both theoreticians and in other applications, dreaming of, and/or working on, such important direction, that would imply quite a revolution not just in drug discovery

Citation:

Diego Liberati (2018) Drug discovery: science and/or art?. J Pharmacol Pharm Res Volume 1(1): 1-1. DOI: 10.31038/JPPR.1000102 